\title{
Tidal gravity observations at Mt. Etna and Stromboli: results concerning the modeled and observed tidal factors
}

\author{
Stefano Panepinto $\left({ }^{1}\right)\left({ }^{3}\right)$, Filippo Greco $\left({ }^{2}\right)$, Dario Luzio $\left({ }^{3}\right)$ and Bernard Ducarme $\left({ }^{4}\right)$ \\ $\left({ }^{1}\right)$ Royal Observatory of Belgium, Brussels, Belgium \\ ${ }^{(2)}$ Istituto Nazionale di Geofisica e Vulcanologia, Sezione di Catania, Italy \\ $\left(^{3}\right)$ Dipartimento di Chimica e Fisica della Terra ed Applicazioni alle Georisorse e ai Rischi Naturali (CFTA), \\ Università degli Studi di Palermo, Italy \\ ${ }^{(4)}$ Research Associate NFSR, Royal Observatory of Belgium, Brussels, Belgium
}

\begin{abstract}
Continuous gravity observations performed in the last few years, both at Mt. Etna and Stromboli, have prompted the need to improve the tidal analysis in order to acquire the best corrected data for the detection of volcano related signals. On Mt. Etna, the sites are very close to each other and the expected tidal factor differences are negligible. It is thus useful to unify the tidal analysis results of the different data sets in a unique tidal model. This tidal model, which can be independently confirmed by a modeling of the tidal parameters based on the elastic response of the Earth to tidal forces and the computation of the ocean tides effects on gravity, is very useful for the precise tidal gravity prediction required by absolute or relative discrete gravity measurements. The change in time of the gravimeters' sensitivity is also an important issue to be checked since it affects not only the results of tidal analysis but also the accuracy of the observed gravity changes. Conversely, if a good tidal model is available, the sensitivity variations can be accurately reconstructed so as to retune observed tidal records with the synthetic tide, since the tidal parameters are assumed to be constant at a given location.
\end{abstract}

Key words tidal gravity models - Mt. Etna - LaCoste and Romberg gravimeters - tidal loading

\section{Introduction}

Continuous gravity recordings in volcanic areas, coupled with continuous GPS observations, are considered a good detection tool of the mass changes linked to magma transfer processes and, thus, to recognize forerunners of paroxysmal vol-

Mailing address: Dr. Stefano Panepinto, Dipartimento di Chimica e Fisica della Terra ed Applicazioni alle Georisorse e ai Rischi Naturali (CFTA), Università degli Studi di Palermo, via Archirafi 36, 90123 Palermo, Italy; e-mail: stefanopanepinto@unipa.it canic events. This geophysical methodology plays a fundamental role in monitoring active volcanoes and predicting eruptive events too $(\mathrm{Ca}-$ macho et al., 2007; Carbone and Greco, 2007).

Spring gravity meters are still the most widely used instruments for microgravity studies in volcanic areas due to their relatively low cost and small size, which make them easy to transport and install. Although spring gravimeters are severely affected by meteorological effects (Warburton and Goodkind, 1977; El Wahabi et al., 1997, 2001; Carbone et al., 2003; Panepinto et al., 2006) and suffer strong instrumental drift compared to superconducting ones, these instruments are also suitable for continuous gravity recording.

The main focus of continuous gravity observations on an active volcano is the detection of 


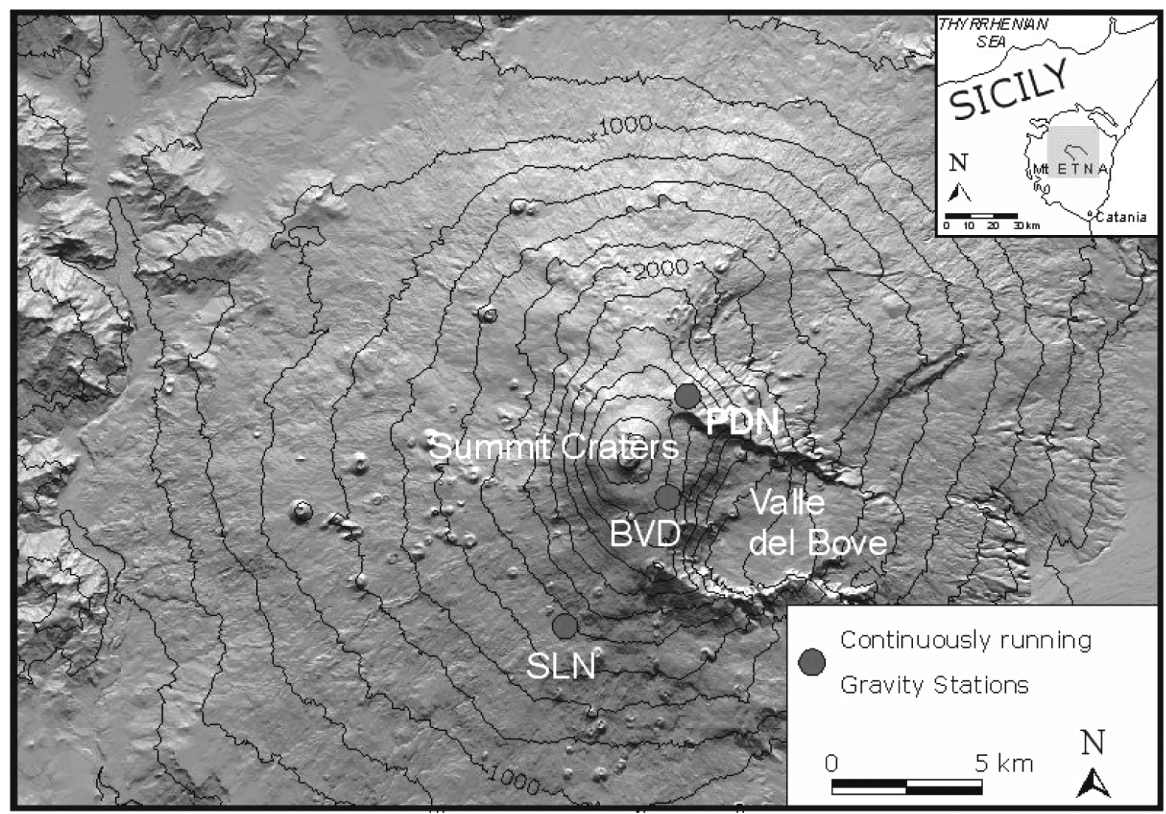

Fig. 1. Sketch map of Mt Etna showing the continuously running gravity stations managed by INGV Catania section.

gravity changes associated with volcanic events with a wide range of evolution rates (periods ranging from minutes to months). However, for periods longer than $10 \mathrm{~h}$, the tidal gravity variations will mask other geophysical phenomena. It is thus very important to efficiently remove the tidal signal, possibly on line. A precise tidal prediction model is thus required. Such a model is also necessary for relative field gravity measurements on volcanoes and the precision requirements are much more demanding if absolute measurements are considered. A first interest of tidal gravity observations on volcanoes is thus to build up an experimental model that can be compared with a modeling of the tidal parameters based on the elastic response of the Earth to tidal forces and the computation of the ocean tides effects on gravity (Section 4).

Two main instrumental problems have to be addressed in order to obtain coherent results using spring gravimeters and especially LaCoste $\&$ Romberg instruments: the adjustment of the scale factor (Section 5) and the changes in sen- sitivity (section 3 ). The maker provides a calibration constant but, when recording the very tiny (less than $0.3 \mathrm{mgal}$ ) tidal changes, one should generally apply a scale factor or normalization constant. This quantity can be determined either by recording side by side with well calibrated instruments (Wenzel et al., 1991; Ducarme and Somerhausen, 1997) or by checking the scale factor on specially designed short baselines e.g., the Hannover vertical base line (Kangieser and Torge, 1981; Timmen and Wenzel, 1994).

On the other hand, spring gravimeters often show sensitivity variations in the order of a few percent (van Ruymbeke, 1998), due for example to ground tilting or temperature effects on the electronics. It is thus necessary to continuously monitor the instrumental sensitivity in order to maintain accurate information concerning real gravity changes.

This paper will chiefly address these two instrumental problems as well as the determination of the best tidal prediction model. These 
steps are a priori conditions to achieve useful gravity recording for the monitoring of volcanic activity.

The observations on Mt. Etna were performed over an interval of about fifteen years. The sequences analyzed are not continuous and were recorded using both LaCoste and Romberg (LCR) and Scintrex spring gravimeters, located (fig. 1): i) about $10 \mathrm{~km}$ south of the active craters at Serra la Nave Astrophysical Observatory (SLN; $1740 \mathrm{~m}$ a.s.1.); ii) $2 \mathrm{~km}$ north-east of the summit NE crater at the Pizzi Deneri Volcanological Observatory (PDN; $2820 \mathrm{~m}$ a.s.1.) and iii) about $1 \mathrm{~km}$ south of the summit SE Crater at the Belvedere site (BVD; $2910 \mathrm{~m}$ a.s.1.). The Stromboli gravimeter (fig. 2 ) is instead located on the slope NE of the volcano edifice at the "Marina di S. Vincenzo» Observatory (STR, $200 \mathrm{~m}$ a.s.l.).

The SLN station $\left(\phi=37.6930^{\circ} \mathrm{N}, \quad \lambda=\right.$ $\left.14.9747^{\circ} \mathrm{E}, h=1740 \mathrm{~m}\right)$, was the first continuous gravity station established on Mt Etna (El Wahabi et al, 1997; Budetta et al., 2000). The observa- tions acquired in this station were performed both with a LCR G-8 (managed by the Royal Observatory of Belgium) between 1992 and 1995, and recently with a Scintrex CG-3M (managed by the Istituto Nazionale di Geofisica e Vulcanologia Catania).

The PDN station $\left(\phi=37.7643^{\circ} \mathrm{N}, \lambda=\right.$ $15.0154^{\circ} \mathrm{E}, h=2820 \mathrm{~m}$ ) is one of the two stations which are very close to the active craters. Observations in this station were performed with the gravimeters LCR D-185 and LCR PET-1081. The BVD station $\left(\phi=37.7408^{\circ} \mathrm{N}, \lambda=15.0091^{\circ} \mathrm{E}\right.$, $h=2910 \mathrm{~m}$ ) is equipped with a LCR D-185. Finally, the STR station $\left(\phi=38.80^{\circ} \mathrm{N}, \lambda=15.2270^{\circ} \mathrm{E}\right.$, $h=200 \mathrm{~m}$ ) is the only one located at Stromboli and the gravity meter for the recordings is the LCR D-157.

Besides gravity, other parameters were acquired in all continuous running gravity stations: instrument tilt in two perpendicular directions, atmospheric temperature, pressure, humidity and voltage from the power system feeding the station. All the gravimeters are equipped

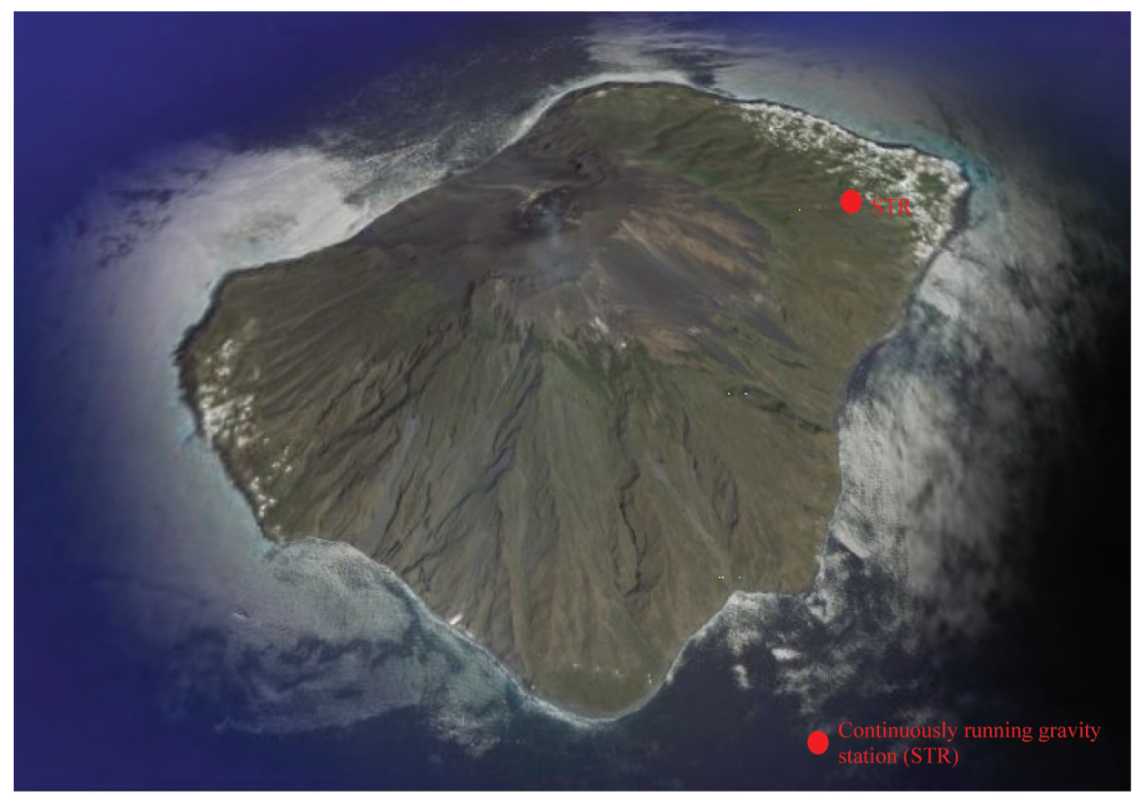

Fig. 2. Satellite picture of Stromboli island showing the location of the continuously running gravity station managed by INGV Catania section $\left(\right.$ google $\left.^{\circledR}\right)$. 
with a feedback system. The resolution is better than $0.1 \mu \mathrm{gal}$ for the LCR instruments while the output of the SCINTREX CG3M is rounded up to the $\mu$ gal unit. Data were recorded at 1da$\mathrm{tum} / \mathrm{min}$ sample rate (each datum is the average calculated over 60 measurements) through a CR10X Campbell Scientific data-logger (A/D bits: 13) (for more details see the website http://www.campbellsci.com/cr10x/).

\section{Tidal analysis procedure}

Tidal analysis determines, for the main tidal waves, the amplitude ratio between the observed tidal amplitude and the theoretical amplitude of the astronomical tide as well as the phase difference between the observed and the theoretical tidal vectors. These quantities are called tidal gravimetric factors (section 4). Furthermore, the analysis provides a tidal model for gravity data reduction retuned with local observations and, in such a way, improves the reliability of the gravity residuals for the detection of volcano related signals.

The preprocessing of the available data sets is based on the «remove-restore» principle and accomplished using the interactive « $T$-soft» software (Van Camp and Vauterin, 2005). A model of tides (tidal prediction) and pressure effects are subtracted from the observations («remove» procedure) and the corrections directly applied on the residues. Anomalous signals such as spikes, steps and large-amplitude oscillations caused by large earthquakes are carefully removed by an interactive procedure. Interpolation of missing data, due to short power interruption, becomes a simple linear interpolation between the two edges of a gap. Corrected observations are then recomputed ( $«$ restore» procedure). The quality of the tidal model is essential, as the interpolated data will in fact be replaced by this model during the «restore» procedure. Very often, poor quality data are kept to avoid the creation of gaps. For the same reason, gaps of various extensions are interpolated, which introduces a non-Gaussian non-stationary noise (Ducarme et al., 2004). Indeed, gaps can introduce systematic errors by leakage, but inappropriate interpolations can be misleading too. Thus, the data are inevitably subjected to various perturbations, including interruptions of the recordings. Completely automated procedures such as the PRETERNA program (Wenzel, 1994) can be dangerous. This is why the «T-soft» software has been developed with a high degree of interactivity.

Data are then decimated to 1-hour sampling for a classical tidal analysis using the ETERNA3.4 software (Wenzel, 1998). The tidal analysis was performed on the data sets acquired both at Etna and Stromboli. The series have different time spans and were performed with different gravity sensors. As emphasized before, the calibration of different gravimeters is not always coherent. On Etna we have thus scaled all the instruments directly on the best data set, the records of LCR G-8 in Serra la Nave. The scale factor of this instrument had been previously determined in the reference station of Brussels (Melchior, 1994). This normalization will be discussed in Section 5. On Stromboli it was difficult to obtain a reliable model to scale the gravimeter (Section 6).

\section{Calibration of the LCR gravimeters and sensitivity variations}

The change in time of the calibration is an important issue to be checked since it affects not only the quality of the data available but also the accuracy of the tidal analysis results and the observed gravity changes. The goal of any tidal measurements is to determine the response of the Earth to the tidal force $F(t)$ through an instrument, using a modeling system. In the output $O(t)$ we cannot separate which response in the physical system is due to the Earth and which to the instrument. Doing a calibration means determining independently the transfer function of the instrument in amplitude as well as in phase. In the Tidal bands the phase behavior of LCR gravimeter equipped with a feedback electronics can be assimilated to a constant time lag, close to $30 \mathrm{~s}$. The maker performs an initial absolute calibration, providing a dial calibration constant $K$ that expresses the force applied to the beam of the gravimeter by a unit rotation of the dial and is usually ex- 
pressed in $\mu \mathrm{gal} /($ dial division). On the other hand, we have to transform the output of the datalogger into equivalent acceleration applied to the beam, i.e. determine the scale calibration $C$ in [physical units] per [recording units]. The usual way of calibrating an LCR instrument is to turn the micrometric screw of $n$ divisions in order to apply a force with a known magnitude $n K$. If the amplitude of the response of the instrument is $l$, the calibration factor of the instrument $C$ is

$$
C=n K / l=K / d
$$

$C$ is expressed in [physical units ( $\mu$ gal)] per [recording units] and $d=l / n$ is the reaction of the instrument per one division of the micrometer. Let us call this procedure «physical calibration». The original observations should be multiplied by $C$ prior to tidal analysis.

The instrumental sensitivity $s$, inverse of the calibration, is then given by

$$
s=l / C=d / K
$$

expressed in [recording units] per [physical units]. Unfortunately, the calibration of a LCR gravimeter equipped with an electronic feedback shows fluctuations of a few per cent (van Ruymbeke, 1998). As the «physical calibration» of an instrument is generally a long and tedious procedure and, what is worse, perturbs the tidal records, there are only a few calibrations avail-

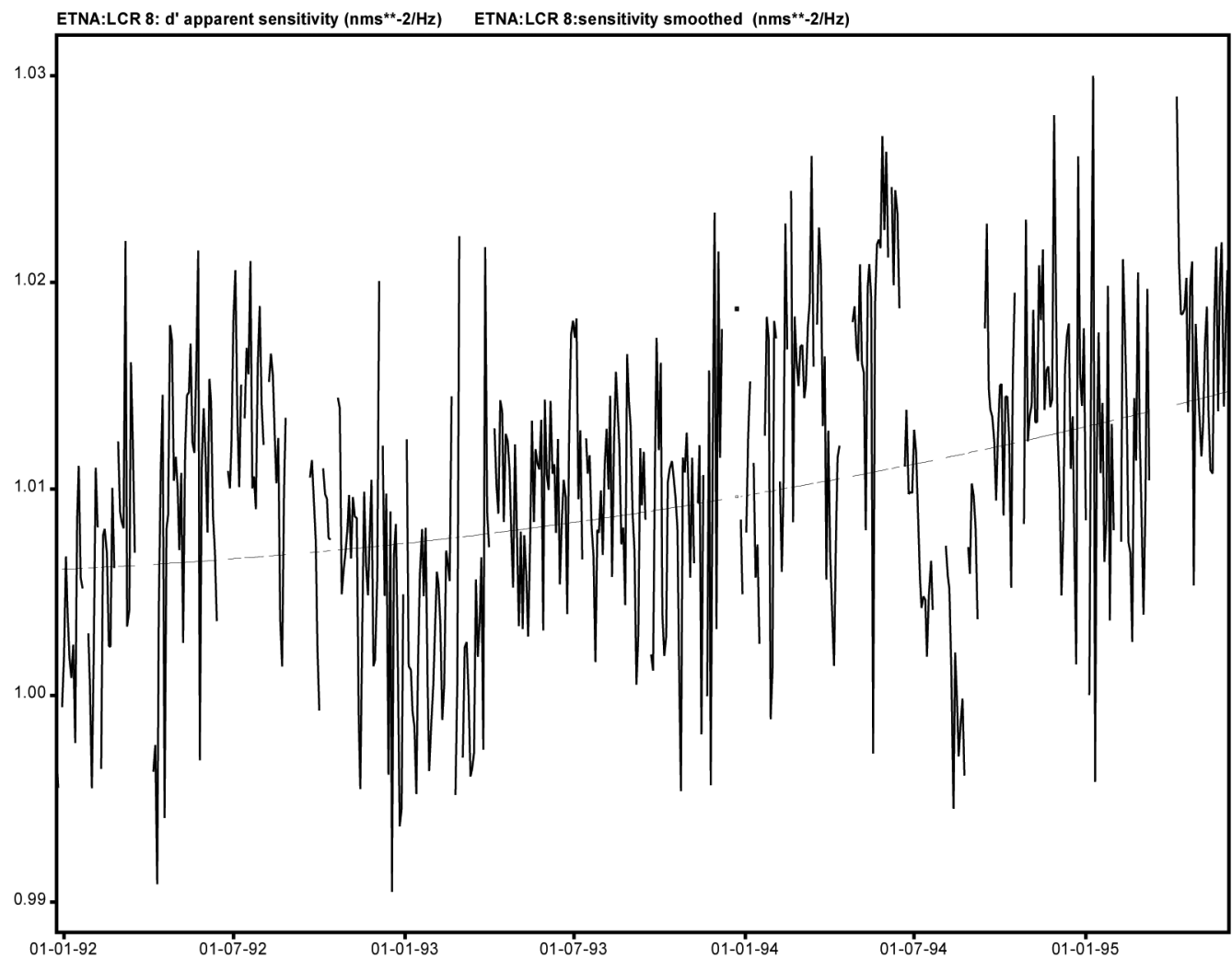

Fig. 3. Sensitivity changes of the LCR G- 8 computed on 48 h blocks. Bold line: sensitivity $s^{\prime}$; thin line: smoothed sensitivity $\hat{s}^{\prime}$. Mean sensitivity is changing of changes by $1 \%$, with seasonal oscillation of $\pm 1.5 \%$. 
able during the recording period and the sensitivity behavior between two calibrations is unknown. The best we can do is to compute an instantaneous calibration value by linear interpolation between two successive calibrations. Fortunately, we can accurately follow the changes in sensitivity using the tidal records themselves as we suppose that the tidal parameters are perfectly stable. If we have a good model for the tidal amplitude factors and phase differences, we can generate a tidal prediction in physical units and fit the observations on the prediction with a given window size e.g., $48 \mathrm{~h}$ (Ducarme, 1970). The regression coefficient $s$, expressed in [recording units] times [physical units], can be called «apparent sensitivity». If the tidal model and the calibration $\mathrm{C}$ are correct and if the sensitivity is perfectly stable we should have always $s^{\prime}=s$.

An option has been implemented in $« T$ soft» under «moving window regression», providing a sequence of apparent sensitivity values $s_{j}^{\prime}$ for every block $j$ (fig. 3). Auxiliary channels, such as pressure can be incorporated in a multilinear regression. We can thus follow the sensitivity variations from block to block. Due to noise and perturbations, it is generally necessary to smooth the individual values $s_{j}^{\prime}$ to obtain a sequence $\hat{s}_{j}^{\prime}$ with a continuous behavior. As an example, we show in fig. 3 the sensitivity changes in the LCR G-8 throughout the acquisition period. The apparent sensitivity increased by $1 \%$ in three and half years. By the proposed procedure we may obtain a general overview on the behavior of the gravimeter. Furthermore, it seems a good tool to detect strong instrumental perturbation during any ongoing volcanic activity and avoid confusion between purely instrumental effects and geophysical ones.

Going one step further, it is possible to remedy the scarcity of «physical calibrations». As the tidal model does not perfectly fit the observations one generally has for block $j$, where a calibration was performed with a value $C_{j}=1 / s_{j}$,

$$
s_{j} \neq \hat{s}_{j}^{\prime}
$$

Let us consider the ratio

$$
f_{j}=\hat{s}_{j}^{\prime} / s_{j}
$$

Due to the noise we have to determine a mean value $\bar{f}$ and its standard deviation by averaging all the $f_{j}$ values of the blocks where a calibration has been physically performed. Considering that the real sensitivity is provided by the «physical calibration», we can estimate an «interpolated calibration» $\breve{C}_{k}$ on each block $k$

$$
\check{C}_{k}=\bar{f} / \hat{s}_{k}^{\prime}
$$

We can linearly interpolate between the slowly changing values of $\breve{C}_{k}$ and multiply each observation by the corresponding value.

\section{Modeling the tidal parameters}

For the main tidal waves (fig. 4), we determine the amplitude $A$ and the phase difference $\alpha$ with respect to the astronomical tide, i.e. the vector $\boldsymbol{A}(A, \alpha)$. The amplitude factor $\delta_{\text {o }}$ (the subscripts mean: $\mathrm{o}=$ observed; th=theoretical; model=computed from the DDW99 model; m=

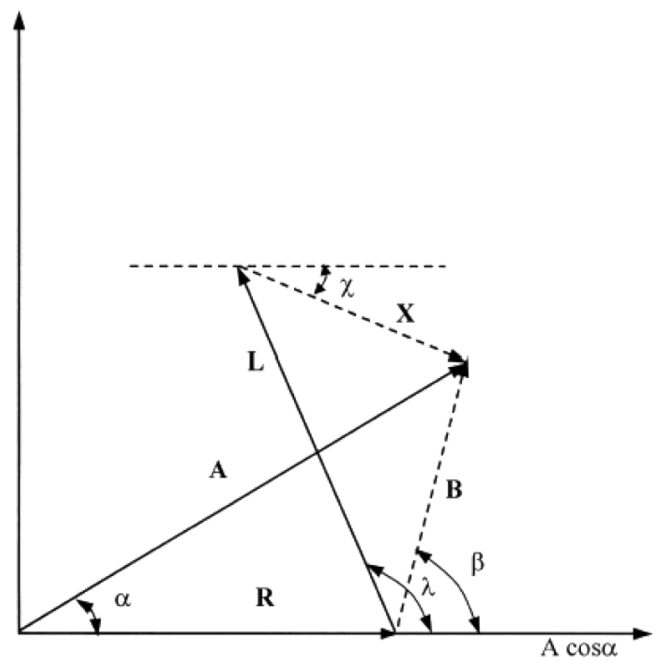

Fig. 4. Phasor plot of a given tidal frequency showing the relationship between the observed tidal amplitude vector $\boldsymbol{A}(A, \alpha)$, the Earth model $\boldsymbol{R}(R, 0)$, the computed ocean tides load vector $\boldsymbol{L}(L, \lambda)$, the tidal residue $\boldsymbol{B}(B, \beta)$ and the corrected residue $\boldsymbol{X}(X, \chi)$, after Melchior (1995). See the text for further explanation. 
modeled) is defined with respect to the theoretical tidal amplitude $A_{\text {th }}$ as the ratio $A / A_{\text {th }}$ (Melchior, 1978). We build the modeled tidal factors based on the body tide amplitude $\boldsymbol{R}\left(A_{\text {th }} \delta_{\text {model }}, 0\right)$, where $\delta_{\text {model }}$ is the amplitude factor computed using a model of the response of the Earth to the tidal force, and the ocean load vector $\boldsymbol{L}(L, \lambda)$ computed from different ocean tides models.

The modeled vector $\boldsymbol{A}_{\mathrm{m}}\left(A_{\mathrm{m}}, \alpha_{\mathrm{m}}\right)$ is given as

$$
A_{m}=R+L
$$

The modeled amplitude factor $\delta_{\mathrm{m}}$ is simply given by the ratio $A_{\mathrm{m}} / A_{\mathrm{th}}$.

The $\boldsymbol{R}$ vector depends on the choice of the body tides model describing the response of the Earth to the tidal forces. Dehant et al. (1999) proposed two different models: the elastic one and the non-hydrostatic/anelastic one. The discrepancy between the elastic and anelastic models is at the level of $0.15 \%$. On the grounds of previous comparisons between the two models (Baker and Bos, 2003, Ducarme et al., 2006), based on the superconducting gravimeter array (Global Geodynamics Project, Crossley et al., 1999), we decided to use the DDW99 non-hydrostatic/anelastic model (DDW99/NH) as a standard.

In such a way the modeled tidal factors are defined as

$$
\boldsymbol{A}_{\mathrm{m}}\left(\delta_{\mathrm{m}} A_{\mathrm{th}}, \alpha_{\mathrm{m}}\right)=\boldsymbol{R}\left(\delta_{\text {model }} A_{\mathrm{th}}, 0\right)+\boldsymbol{L}(L, \lambda)
$$

We also built the residual vector $\mathbf{B}(B, \beta)$ and the final residue $\mathbf{X}(X, \chi)$ :

$$
\begin{gathered}
\boldsymbol{B}(B, \beta)=\boldsymbol{A}\left(A_{\mathrm{th}} \delta_{o}, \alpha\right)-\boldsymbol{R}(R, 0) \\
X(X, \chi)=\boldsymbol{B}(B, \beta)-\boldsymbol{L}(L, \lambda) .
\end{gathered}
$$

We can directly compare the vectors $\boldsymbol{A}$ and $\boldsymbol{A}_{\mathrm{m}}$ to evaluate the adequacy of the corresponding ocean tides model.

As early as 1979, Schwiderski constructed ocean tide models (SCW80; Schwiderski, 1980) using the hydrodynamic interpolation method and introducing tide gauge data on coast lines and islands. For the first time, he provided a relatively complete and basic ocean tidal model for loading correction in geodesy and geophysics. Since
1994, a series of new ocean tidal models has been developed based mainly on the Topex/Poseidon (T/P) satellite altimeter data. The first generation of models we consider here are: CSR3 (Eanes and Bettadpur, 1996), FES95 (Le Provost et al., 1994) and ORI96 (Matsumoto et al., 1995). These models were extensively tested in Shum et al. (1997). Most of the more recent ones used subsequently represent updates of the previous ones: CSR4, NAO99 (Matsumoto et al., 2000), GOT00 (Ray, 1999), FES02 and TPX06. An important difference between models is the grid size which has been progressively refined from $\left(1^{\circ} \times 1^{\circ}\right)$ for $\mathrm{SCW} 80$, to $\left(0.5^{\circ} \times 0.5^{\circ}\right)$ for CSR3, CSR4, FES95, GOT00, NAO99, ORI96 and finally $\left(0.25^{\circ} \times 0.25^{\circ}\right)$ for FES02 and TPX06. As a result the approximation of the real shape of the coast was steadily improved. A second improvement concerns the global water mass balance during one tidal cycle. It is never perfectly achieved but has also been continuously improved.

The tidal loading vector $\boldsymbol{L}$ was evaluated by performing a convolution integral between the ocean tide models and the load Green's function computed by Farrell (1972). The Green's functions are tabulated according to the angular distance between the station and the load. The water mass is condensed at the centre of each cell and the Green's function is interpolated according to the angular distance. This computation is rather delicate for coastal stations and models computed on a coarse grid, as the station can be located very close to the centre of the cell. The numerical effect can be largely overestimated. To avoid this problem our tidal loading computation checks the position of the station with respect to the centre of the grid. If the station is located inside the cell, this cell is eliminated from the integration and the result is considered not reliable (Melchior et al., 1980). For the first generation of models, the effect of the imperfect mass conservation is corrected on the basis of the code developed by Moens (Melchior et al., 1980). Following Zahran's (2000) suggestion, we also computed mean tidal loadings for different combinations of models.

Table I presents the tidal factors modeled using the DDW99/NH model (Dehant et al., 1999) and nine different ocean tide models for the SLN station. In this comparison we restrict 
Table I. Modeled tidal factors $\left(\delta_{m}, \alpha_{m}\right)$ computed using the DDW99/NH model and nine different ocean tides models for the SLN station compared with LCR G-8 results $\left(\delta_{o}, \alpha_{o}\right)$. We consider the two main diurnal tidal waves $\left(O_{1}\right.$ : lunar declinational, $K_{1}$ : luni-solar declinational) and the main semidiurnal one ( $M_{2}$ : lunar principal). $\sigma_{\delta}$ and $\sigma_{\alpha}$ are the standard deviations associated with the mean of the nine models.

\begin{tabular}{|c|c|c|c|c|c|c|c|}
\hline Wave & $O_{1}$ & $K_{1}$ & $M_{2}$ & $\delta_{M 2} / \delta_{O 1}$ & & & \\
\hline $\begin{array}{c}\text { Theoretical } \\
\text { amplitude ( } \mu \text { gal })\end{array}$ & 30.058 & 42.274 & 47.031 & & & & \\
\hline $\begin{array}{l}\text { Modeled } \\
\text { SLN8 }\end{array}$ & $\delta_{m}$ & $\begin{array}{c}\alpha_{m} \\
(\operatorname{deg})\end{array}$ & $\delta_{m}$ & $\begin{array}{c}\alpha_{m} \\
\text { (deg) }\end{array}$ & $\delta_{m}$ & $\begin{array}{c}\alpha_{m} \\
\text { (deg) }\end{array}$ & \\
\hline 1. SCW80 & 1.1502 & -0.006 & 1.1329 & 0.110 & 1.1749 & 0.936 & 1.0215 \\
\hline 2. ORI96 & 1.1497 & 0.169 & 1.1333 & 0.323 & 1.1712 & 0.994 & 1.0187 \\
\hline 3. CSR3 & 1.1492 & 0.000 & 1.1310 & 0.307 & 1.1742 & 1.043 & 1.0218 \\
\hline 4. FES95 & 1.1519 & 0.091 & 1.1342 & 0.229 & 1.1729 & 1.010 & 1.0182 \\
\hline Mean 1-4 & 1.1502 & 0.063 & 1.1328 & 0.242 & 1.1733 & 0.996 & 1.0200 \\
\hline 5 FES02 & 1.1482 & 0.097 & 1.1346 & 0.128 & 1.1747 & 1.028 & 1.0231 \\
\hline 6 CSR4 & 1.1521 & 0.098 & 1.1328 & 0.259 & 1.1759 & 1.155 & 1.0207 \\
\hline 7 GOT00 & 1.1510 & 0.076 & 1.1343 & 0.185 & 1.1744 & 1.056 & 1.0203 \\
\hline 8 NAO99 & 1.1511 & 0.171 & 1.1345 & 0.240 & 1.1735 & 1.017 & 1.0195 \\
\hline 9 TPX06 & 1.1510 & 0.149 & 1.1341 & 0.190 & 1.1748 & 0.924 & 1.0207 \\
\hline Mean 5-9 & 1.1507 & 0.118 & 1.1341 & 0.200 & 1.1747 & 1.036 & 1.0209 \\
\hline Global mean & 1.1505 & 0.094 & 1.1335 & 0.219 & 1.1741 & 1.018 & 1.0205 \\
\hline \multirow[t]{2}{*}{$\begin{array}{l}\text { Standard deviation } \\
\text { global mean }\end{array}$} & $\sigma_{\delta}$ & $\begin{array}{c}\sigma_{\alpha} \\
(\operatorname{deg})\end{array}$ & $\sigma_{\delta}$ & $\begin{array}{c}\sigma_{\alpha} \\
(\mathrm{deg})\end{array}$ & $\sigma_{\delta}$ & $\begin{array}{c}\sigma_{\alpha} \\
(\mathrm{deg})\end{array}$ & \\
\hline & 0.0013 & 0.065 & 0.0012 & 0.073 & 0.0014 & 0.068 & \\
\hline $\begin{array}{l}\text { Observed } \\
\text { SLN }\end{array}$ & $\delta_{o}$ & $\begin{array}{c}\alpha_{o} \\
\text { (deg) }\end{array}$ & $\delta_{o}$ & $\begin{array}{c}\alpha_{o} \\
(\mathrm{deg})\end{array}$ & $\delta_{o}$ & $\begin{array}{c}\alpha_{o} \\
(\mathrm{deg})\end{array}$ & \\
\hline $\begin{array}{c}\text { (LCR G-8) } \\
22 / 12 / 1991 \\
-03 / 06 / 1995\end{array}$ & 1.1499 & 0.116 & 1.1336 & 0.127 & 1.1742 & 1.028 & 1.0211 \\
\hline $\begin{array}{c}\text { Error } \\
f_{N} \text { factor }\end{array}$ & $\begin{array}{c} \pm .0005 \\
0.989975\end{array}$ & \pm .026 & \pm .0004 & \pm .018 & \pm .0003 & \pm .012 & \pm .0010 \\
\hline
\end{tabular}

ourselves to the three main tidal waves: two main diurnal tidal components $\left(O_{1}\right.$ lunar declinational wave, period $25 \mathrm{~h} 49 \mathrm{~min}$; $K_{1}$ luni-solar declinational wave, period $23 \mathrm{~h} 56 \mathrm{~min}$ ) and the main semidiurnal component $\left(M_{2}\right.$ Lunar principal wave, period $12 \mathrm{~h} 25 \mathrm{~min}$ ).

We can consider two groups of models, the older models until 1996 (SCW80, ORI96, CSR3, FES95) on the one hand, and the new generation of models (FES02, CSR4, GOTOO, NA099 and TPX06) on the other. Mean modeled tidal amplitude factors and phase differences are presented in table I for the older models (mean 1-4), for the models of second generation (mean 5-9) and for all the models (global). The standard deviations on the mean of the 9 models are close to $0.1 \%$ for the amplitude factors and $0.07^{\circ}$ on the phase difference. The 
Table II. Final residues $\boldsymbol{X}$ at SLN, computed using the DDW99/NH model and nine different ocean tides models (instrument LCR G-8).

\begin{tabular}{ccccccc}
\hline \hline Model & $O_{1}$ & $K_{1}$ & $M_{2}$ & & & \\
\hline $\begin{array}{c}\text { Theoretical } \\
\text { amplitude }(\mu \text { gal })\end{array}$ & 30.058 & 42.274 & 47.031 & & & \\
\hline SLN8 & & & & & & \\
\hline & $(\mu$ gal $)$ & $\begin{array}{c}\chi \\
(\mathrm{deg})\end{array}$ & $\begin{array}{c}X \\
(\mu \mathrm{gal})\end{array}$ & $\begin{array}{c}\chi \\
(\mathrm{deg})\end{array}$ & $\begin{array}{c}X \\
(\mu \mathrm{gal})\end{array}$ & $\begin{array}{c}\chi \\
\text { deg })\end{array}$ \\
\hline 1. SCW80 & 0.074 & 97.84 & 0.036 & 23.63 & 0.094 & 111.02 \\
2. ORI96 & 0.033 & -77.75 & 0.164 & -84.17 & 0.146 & 13.89 \\
3. CSR3 & 0.074 & 72.12 & 0.188 & -72.90 & 0.015 & -80.20 \\
4. FES95 & 0.061 & 165.66 & 0.0 .088 & -105.09 & 0.066 & 16.02 \\
Mean 1-4 & 0.033 & 107.48 & 0.102 & -69.95 & 0.054 & 35.98 \\
5. FES02 & 0.052 & 12.47 & 0.041 & -178.23 & 0.021 & -178.07 \\
6. CSR4 & 0.067 & 170.82 & 0.117 & -71.25 & 0.145 & -121.15 \\
7. GOT00 & 0.041 & 143.31 & 0.055 & -118.59 & 0.029 & -107.52 \\
8. NAO99 & 0.050 & -137.61 & 0.101 & -110.53 & 0.036 & 18.94 \\
9. TPX06 & 0.039 & -148.80 & 0.057 & -111.72 & 0.103 & 104.76 \\
Mean 5-9 & 0.024 & -176.48 & 0.064 & -105.71 & 0.021 & -157.16 \\
\hline
\end{tabular}

SLN: modelled and observed tidal vectors

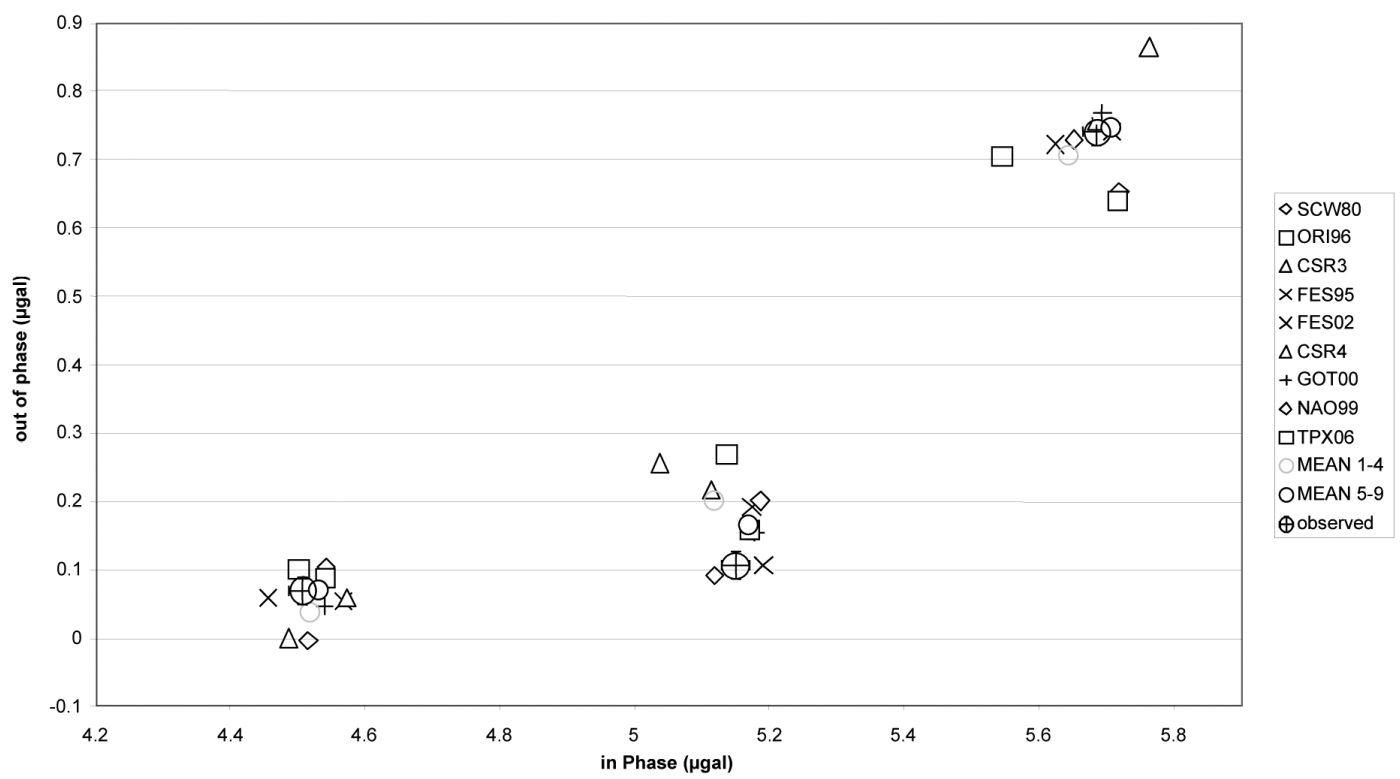

Fig. 5. Comparison of the modelled $\boldsymbol{A}_{\boldsymbol{m}}$ and observed $\boldsymbol{A}$ tidal vectors for the wave $O_{1}$. The horizontal scale has been adjusted by subtracting the astronomical amplitude $A_{\text {th }}$. From left to right waves $O_{1}, K_{1}$ and $M_{2}$ White background: 5 recent models, circles mean $1-4$ and 5-9, $\oplus$ observed vector. 
Table III. Observed tidal factors calculated using data obtained at Serra La Nave (SLN), Pizzi Deneri (PDN) and Belvedere (BVD) gravity stations.

\begin{tabular}{|c|c|c|c|c|c|c|c|}
\hline Tidal Component & & $\mathbf{O}_{1}$ & & $\mathbf{K}_{1}$ & \multicolumn{2}{|c|}{$\mathbf{M}_{2}$} & $\delta_{\mathrm{M} 2} / \delta_{\mathrm{O} 1}$ \\
\hline $\begin{array}{c}\text { Theoretical Amplitude } \\
\text { at SLN ( } \mu \text { gal) }\end{array}$ & & 30.058 & & 2.274 & \multicolumn{2}{|c|}{47.031} & \\
\hline OBSERVED & $\delta_{o}$ & $\alpha_{o}(\operatorname{deg})$ & $\delta_{\mathrm{o}}$ & $\alpha_{o}(\operatorname{deg})$ & $\delta_{o}$ & $\alpha_{\mathrm{o}}(\mathrm{deg})$ & \\
\hline $\begin{array}{c}\text { SLN station } \\
\text { (LCR G-8) } \\
22 / 12 / 1991 \\
03 / 06 / 1995\end{array}$ & 1.1499 & 0.116 & 1.1336 & 0.127 & 1.1742 & 1.028 & 1.0211 \\
\hline Error & \pm .0005 & \pm .026 & \pm .0004 & \pm .018 & \pm .0003 & \pm .012 & \pm .0010 \\
\hline$f_{N}$ factor & $0.98975^{*}$ & & & & & & \\
\hline $\begin{array}{c}\text { SLN station } \\
\text { (Scintrex CG-3M) } \\
30 / 11 / 2002 \\
13 / 07 / 2003 \\
\end{array}$ & 1.1487 & -0.023 & 1.1355 & 0.053 & 1.1744 & 0.876 & 1.0224 \\
\hline Error & \pm .0009 & \pm .044 & \pm .0007 & \pm .033 & \pm .0008 & \pm .040 & \pm .0012 \\
\hline$f_{N}$ factor & $1 * *$ & & & & & & \\
\hline $\begin{array}{c}\text { PDN station } \\
\text { (LCR D-185) } \\
01 / 11 / 2002 \\
30 / 12 / 2002\end{array}$ & 1.1405 & -0.459 & 1.1263 & 0.192 & 1.1737 & 0.572 & 1.0291 \\
\hline Error & \pm .0033 & \pm .167 & \pm .0020 & \pm .101 & \pm .0019 & \pm .092 & \pm .0038 \\
\hline$f_{N}$ factor & 0.9974 & & & & & & \\
\hline $\begin{array}{c}\text { PDN station } \\
\text { (LCR PET-1081) } \\
01 / 06 / 2005 \\
31 / 12 / 2005\end{array}$ & 1.1489 & 0.056 & 1.1368 & -0.117 & 1.1746 & 0.807 & 1.0224 \\
\hline Error & \pm .0014 & \pm .069 & \pm .0013 & \pm .057 & \pm .0007 & \pm .033 & \pm .0017 \\
\hline$f_{N}$ factor & 0.9867 & & & & & & \\
\hline $\begin{array}{c}\text { BVD station } \\
\text { (LCR D-185) } \\
01 / 05 / 2005 \\
31 / 12 / 2005 \\
\end{array}$ & 1.1543 & 0.221 & 1.1381 & 0.378 & 1.1755 & 0.755 & 1.0184 \\
\hline Error & \pm .0043 & \pm .212 & \pm .0033 & \pm .209 & \pm .0019 & \pm .091 & \pm .0047 \\
\hline$f_{N}$ factor & 0.9974 & & & & & & \\
\hline $\begin{array}{c}\text { BVD station } \\
\text { (LCR D-185) } \\
\text { 19/03/2005 } \\
31 / 01 / 2006\end{array}$ & 1.1524 & 0.413 & 1.1289 & -0.711 & 1.1744 & 0.707 & 1.0191 \\
\hline Error & \pm .0052 & \pm .258 & \pm .0039 & \pm .214 & \pm .0018 & \pm .088 & \pm .0055 \\
\hline$f_{N}$ factor & 0.9974 & & & & & & \\
\hline $\begin{array}{c}\text { BVD station } \\
\text { (LCR D-185) } \\
\text { 21/11/2004 } \\
\text { 31/01/2006 } \\
\end{array}$ & 1.1502 & -0.093 & 1.1283 & -0.621 & 1.1739 & 0.753 & 1.0206 \\
\hline Error & \pm .0052 & \pm .259 & \pm .0040 & \pm .218 & \pm .0019 & \pm .091 & \pm .0055 \\
\hline$f_{N}$ factor & 0.9974 & & & & & & \\
\hline
\end{tabular}

*Brussels reference station (Melchior, 1994)

** no normalization required, see text. 
precision on the mean should be 3 times better.

The observations made with LCR G-8 have the highest internal precision (table III) and the scale factor of this instrument was previously adjusted in Brussels (Melchior, 1994). This instrument is thus a good reference to appreciate the validity of the modeled factors. The observed amplitude factors $\delta_{0}$ are in agreement with the mean modeled ones $\delta_{\mathrm{m}}$ within the associated errors. The calibration is thus confirmed. The ratio $\delta_{\mathrm{M}_{2}} / \delta_{O_{1}}$, which is independent from the calibration, perfectly fits the mean of the 5 recent models (table I). Figure 5 indicates clearly that the mean of the 5 recent models (white circles) is closest to the observations $(\oplus)$. The residual vector $\boldsymbol{X}$ is a measure of the global misfit between the observed vector $\boldsymbol{A}$ and the modeled one $\boldsymbol{A}_{\mathrm{m}}$ (fig. 3). Looking at table II, we see that the amplitude of the final residues $\boldsymbol{X}$ ranges from less than $0.1 \%$ of the wave amplitude for $O_{1}$ and $M_{2}$ to $0.15 \%$ for $K_{1}$, if we consider the mean of the 5 recent models. A tidal prediction based on this mean will thus be correct at the level of $0.3 \mathrm{mgal}$ on a global tidal range close to $260 \mu$ gal. It is not surprising that the recent models are giving the best results, as some of the older models do not include the Mediterranean Sea.

\section{Standardizing the calibration factors in- side the Etna stations}

Using the maker's calibration, the observed tidal factors show differences in the amplitude factor $\delta_{0}$ with respect to the modeled ones. On the one hand, the calibration factor of the instrument influences the «in phase components», and induces only a perturbation in the amplitude factor $\delta_{0}$ of the tidal group considered, while on the other, the electronics of the feedback or the time keeping system influence the «out of phase» components, thereby inducing a difference in the observed phase $\alpha_{0}$.

As we stated before, on Mt. Etna the sites are very close to each other and the differences between the tidal factors should be negligible. We thus applied a different normalization factor $\left(f_{N}\right)$ to each instrument to unify all the observed tidal factors (see table III).

The normalization factor of LCR G-8 was determined from tidal gravity observations performed at «Brussels Fundamental Station» (Melchior, 1994). It was shown in the previous section that the results are in very good agreement with the modeling based on the ocean tides load vector and the DDW99/NH model.

The tidal parameters determined with the SCINTREX agree within the error bars with the LCR G-8. It is thus not necessary to apply a normalization factor to this instrument. The SCINTREX calibration system seems correct and homogeneous as already indicated in other stations (Ducarme and Somerhausen, 1997).

For the other instruments, a normalization factor is computed to obtain the best agreement with the LCR G-8 for the waves $O_{1}$ and $M_{2}$. Taking Into account the amplitude ratio between $O_{1}$ and $M_{2}$, we scale the instruments using the following formula

$$
f_{N}=0.4 \frac{\delta_{G_{-}}\left(O_{1}\right)}{\delta\left(O_{1}\right)}+0.6 \frac{\delta_{G_{-}}\left(M_{2}\right)}{\delta\left(M_{2}\right)}
$$

For LCR D-185 the normalization was computed on the longest available series (21/11/200431/01/2006) obtained in BVD. The same normalization was used in PDN. It should be noted that the scale factors of the different LCR gravimeters are very homogeneous. They differ only by $\pm 0.1 \%$.

Table III compares the results after normalization. The ratio $\delta_{\mathrm{M}_{2}} / \delta_{O_{1}}$ is a very good indicator of the quality of the data. Being independent of the calibration, it should be the same for all the instruments installed on Etna. LCR G-8, PET-1081 and Scintrex CG-3M have indeed very coherent values for $\delta_{\mathrm{M}_{2}} / \delta_{O_{1}}$. It is not the case for LCR D-185 at PDN station. In BVD we also considered shorter data sets bounded by major interruptions. The results on $M_{2}$ are very stable while a strong perturbation affects $K_{1}$ during the second part of 2005. The ratio $\mathrm{M}_{2} / \mathrm{O}_{1}$ is also affected. These facts reflect a strong perturbation in the diurnal band as the meteorological wave $S_{1}$ (period $2 \mathrm{~h}$ ) is very close to the period of $K_{1}(23 \mathrm{~h} 58 \mathrm{~min})$. The stability of the temperature is indeed crucial in that respect. Inside the perturbed portions of the tidal gravity records the interpretation of anomalous signals should be made with caution. 
Table IV. Modeled tidal factors at STR calculated with fine grid ocean tides models: TPX06, FES02 and FES04.

\begin{tabular}{|c|c|c|c|c|c|c|c|}
\hline Model (STR) & \multicolumn{2}{|c|}{$O_{1}$} & \multicolumn{2}{|c|}{$K_{1}$} & \multicolumn{2}{|c|}{$M_{2}$} & $\delta_{M 2} / \delta_{O 1}$ \\
\hline \multirow{2}{*}{$\begin{array}{c}\text { Theoretical } \\
\text { amplitude ( } \mu \text { gal) }\end{array}$} & \multicolumn{2}{|c|}{30.33} & \multicolumn{2}{|c|}{42.65} & \multicolumn{2}{|c|}{46.61} & \\
\hline & $\delta_{m}$ & $\begin{array}{c}\alpha_{m} \\
(\mathrm{deg})\end{array}$ & $\delta_{m}$ & $\begin{array}{c}\alpha_{m} \\
(\mathrm{deg})\end{array}$ & $\delta_{m}$ & $\begin{array}{c}\alpha_{m} \\
(\mathrm{deg})\end{array}$ & \\
\hline 1 ТРХ06 $(0.25 \times 0.25)$ & 1.1514 & 0.142 & 1.1353 & 0.149 & 1.1762 & 0.647 & 1.0215 \\
\hline 2 FES02 $(0.25 \times 0.25)$ & 1.1491 & 0.124 & 1.1364 & 0.058 & 1.1760 & 0.644 & 1.0234 \\
\hline 3 FES04 $(0.125 \times 0.125)$ & 1.1505 & 0.138 & 1.1379 & 0.011 & 1.1759 & 0.379 & 1.0221 \\
\hline Mean & 1.1503 & 0.135 & 1.1366 & 0.073 & 1.1760 & 0.557 & 1.0223 \\
\hline $\begin{array}{c}\text { Observed (STR) } \\
\text { (LCR D-157) }\end{array}$ & $\delta_{o}$ & $\begin{array}{c}\alpha_{o} \\
(\operatorname{deg})\end{array}$ & $\delta_{o}$ & $\begin{array}{c}\alpha_{o} \\
(\mathrm{deg})\end{array}$ & $\delta_{o}$ & $\begin{array}{c}\alpha_{o} \\
(\mathrm{deg})\end{array}$ & \\
\hline $13 / 05 / 2005-31 / 08 / 2005$ & 1.1503 & 0.013 & 1.1348 & -0.211 & 1.1760 & -0.532 & 1.0223 \\
\hline $\begin{array}{c}\text { Error } \\
f_{N} \text { factor }\end{array}$ & $\begin{array}{c} \pm .0019 \\
0.99083\end{array}$ & \pm .095 & \pm .0013 & \pm .064 & \pm .0006 & \pm .030 & \pm .0020 \\
\hline
\end{tabular}

Table V. Ocean tide loading vector $\boldsymbol{L}$ computed with different ocean tides model. Local: rejected cell only; global: all accepted cells.

\begin{tabular}{ccccccc}
\hline \hline Model (STR) & \multicolumn{2}{c}{$O_{1}$} & \multicolumn{2}{c}{$K_{1}$} & \multicolumn{2}{c}{$M_{2}$} \\
\hline $\begin{array}{c}\text { Theoretical } \\
\text { amplitude }(\mu \mathrm{gal})\end{array}$ & \multicolumn{2}{c}{30.33} & \multicolumn{2}{c}{42.65} & \multicolumn{2}{c}{46.61} \\
& & & & & & \\
\hline & $L(\mu \mathrm{gal})$ & $\lambda(\mathrm{deg})$ & $L(\mu \mathrm{gal})$ & $\lambda(\mathrm{deg})$ & $L(\mu \mathrm{gal})$ & $\lambda(\mathrm{deg})$ \\
\hline TPX06 (0.25x0.25) Global & 0.121 & 134.3 & 0.127 & 83.9 & 0.887 & 43.1 \\
Local & 0.044 & 37.5 & 0.129 & -30.7 & 0.521 & -84.9 \\
FES02 (0.25x0.25) Global & 0.173 & 154.2 & 0.079 & 38.4 & 0.879 & 43.3 \\
Local & 0.067 & 77.2 & 0.178 & -20.3 & 0.571 & -84.4 \\
FES04 (0.125x0.125) Global & 0.142 & 143.8 & 0.125 & 4.37 & 0.729 & 29.14 \\
Local & 0.070 & 55.6 & 0.228 & -28.1 & 0.699 & -84.8 \\
\hline
\end{tabular}

Table VI. Final residues $\boldsymbol{X}$ of the STR station (instrument LCR D-157); $f_{N}=0.99083$. These residues correspond well to a load vector orthogonal to the astronomical tides

\begin{tabular}{ccccccc}
\hline \hline Model (STR) & \multicolumn{2}{c}{$O_{1}$} & \multicolumn{2}{c}{$K_{1}$} & \multicolumn{2}{c}{$M_{2}$} \\
\hline $\begin{array}{c}\text { Theoretical } \\
\text { amplitude }(\mu \text { gal })\end{array}$ & 30.058 & \multicolumn{2}{c}{42.274} & \multicolumn{2}{c}{47.031} \\
& $X(\mu$ gal $)$ & $\lambda($ deg $)$ & $X(\mu$ gal $)$ & $\lambda($ deg $)$ & $X(\mu$ gal $)$ & $\lambda($ deg $)$ \\
\hline 1 TPX06 & 0.09 & -113 & 0.31 & -94 & 1.10 & -90 \\
2 FES02 & 0.08 & -61 & 0.24 & -107 & 1.10 & -90 \\
3 FES04 & 0.08 & -93 & 0.23 & -125 & 0.85 & -90 \\
Mean 1-3 & 0.07 & -90 & 0.25 & -106 & 1.02 & -90 \\
\hline
\end{tabular}




\section{Tidal gravity observations at Stromboli}

The analysis results for the Stromboli station $\left(\phi=38.800^{\circ}, \lambda=15.227^{\circ}\right)$ are shown in table IV. Due to the position of the station on a small island, the modeling of the tidal factors is very difficult as the island is much smaller than the grid size for most of the ocean tide models.

This is why we selected the models with the finest grids, namely either $0.25^{\circ} \times 0.25^{\circ}$ (FES02, TPX06) or even $0.125^{\circ} \times 0.125^{\circ}$ (FES04). When the tidal loading computation program notices that the station is indeed inside the cell and not too close from the grid center, this cell can be divided and the 4 portions reexamined separately. If the station lies outside some of the portions, the corresponding loading and attraction effect can be computed. The procedure is reiterated up to some predefined level (Melchior et al., 1980). For the TPX06 and FES02 models, the cell centered at $\phi=38.75^{\circ}$ and $\lambda=15.25^{\circ}$ is rejected, while for the $0.125^{\circ} \times 0.125^{\circ}$ model the cell at $\phi=38.75^{\circ}$ and $\lambda=15.25^{\circ}$ is subdivided and only the $0.0625^{\circ} \times 0.0625^{\circ}$ square centered at $\phi=38.781^{\circ}$ and $\lambda=15.219^{\circ}$ is rejected.

As the observed ratio $M_{2} / O_{1}$ is very close to the modeled one, normalization based on the results of $O_{1}$ and $M_{2}$ provides amplitude factors which agree with the mean of the 3 models for the diurnal and the semi-diurnal waves (table IV). However, the phase difference $\alpha_{\mathrm{o}}$ is much lower than the models especially for $M_{2}$. It was found that the «local» load vectors, associated with the rejected cells, are out of phase compared with the «global» load vector (table V). As the phase of the local load vector for $M_{2}$ is orthogonal to the astronomical tide, it will only affect the phase of the modeled vector $\boldsymbol{A}_{\mathrm{m}}$. If we consider an un-modeled load close to $0.7 \mu \mathrm{gal}$, orthogonal to the astronomical tide, it will diminish the $M_{2}$ phase of $0.9^{\circ}$, while we observe a diminution of $1^{\circ}$ in table IV.

If we consider the final residues in table VI, we see that they are orthogonal to the astronomical tides with amplitude similar to the «local» load vectors of table V. However, these vectors are not accurate enough and, to refine the modeling of the tidal factors, the exact contours of the Island are required to introduce them into the grid to avoid covering land by ocean. For the while the best model is given by the normalized analysis of LCR D-157, as the phase difference with the modeled factors can be reasonably explained by the influence of the very local water masses neglected during the loading computations.

\section{Conclusions}

Refined tidal analysis requires a long series of observations. From the long series of tidal gravity observations obtained with the LCR G8 , which had been calibrated in the Brussels fundamental station, it was possible to obtain an experimental tidal gravity model, which fits very well a modeling of the tidal parameters based on the elastic response of the Earth to tidal forces and the computation of the ocean tides effects on gravity. As the Etna stations are very close to each other, it was possible to adjust the scale factors of all the other instruments on the same experimental model. The calibration of the LaCoste \& Romberg gravimeters used in this study is now fairly homogeneous and accurate. The Scintrex CG-3M did not require any normalization.

Since the ratio $\delta_{\mathrm{M}_{2}} / \delta_{O_{1}}$ does not depend on the calibration factor of the instrument, we compare the ratios obtained by the observations with the modeled ones. As a result, the values $\delta_{\mathrm{M}_{2}} / \delta_{O_{1}}$ of the LCR G-8, Scintrex CG-3M and LCR PET-1081 are very close to the model value 1.021. Some portions of the records with LCR D-185 are perturbed. It is probably difficult to detect any geophysical signal in the LCR D-185 series.

The observations performed on Stromboli Island highlighted the difficulty of obtaining a correct model from tidal loading computation, as the grid of the best ocean tides models is still too coarse to accurately follow the repartition of the ocean and land masses. Fortunately, the influence of the water masses closest to the station essentially affects the phase of the observations so that a normalization of the gravimeter was still possible.

We also presented a methodology to follow the sensitivity variations of the spring gravimeters when a good tidal model is available. It is 
an important issue as these sensitivity variations affect not only the quality of the available data but also the accuracy of the tidal analysis results and of the observed gravity changes. It also seems a good tool to detect strong instrumental perturbations during any ongoing volcanic activity and avoid confusion between purely instrumental effects and geophysical ones.

The series of normalized gravity residues routinely obtained on Etna and Stromboli could be used to better detect the possible signals due to volcanic activity. Finally, the improved tidal gravity model will be useful for gravity surveys and absolute measurements.

\section{Acknowledgements}

S. Panepinto worked at the Royal Observatory of Belgium (ROB) during six months in the framework of the bilateral collaboration between ROB and Prof. D. Luzio of the University of Palermo, thanks to funding by the Italian Government (grant n. 76202 Università degli Studi di Palermo). This project would not be possible without the collaboration between ROB and the Istituto Nazionale di Geofisica e Vulcanologia, Catania section.

\section{REFERENCES}

BAKER, T.F. and M.S. Bos (2003): Validating Earth and ocean models using tidal gravity measurements, Geophys. J. Int., 152, 468-485.

Budetta, G., D. Carbone and F. Greco (2000): Gravity measurements, in Data Related to Eruptive Activity, Unrest Phenomena and Other Observations on the Italian Active Volcanoes - 1996, edited by L. VILLARI, Acta Vulcanol., 12 (1-2), 86-90.

Camacho, A.G., J. Fernández, M. Charco, K.F. Tiampo and G. Jentzsch (2007): Interpretation of 1992-1994 gravity changes around Mayon volcano, Philippines, using point sources, Pure Appl. Geophys., 733-749, doi: 10.1007/s00024-007-0185-8.

Carbone, D. and F. Greco (2007): Review of microgravity observations at Mt. Etna: a powerful tool to monitor and study active volcanoes, Pure Appl. Geophys., 769790, doi:10.1007/s00024-007-0194-7.

Carbone, D., G. Budetta, F. Greco and H. Rymer (2003). Combined discrete and continuous gravity observations at Mt. Etna, J. Volcanol. Geotherm. Res., 123, 123-135.

Crossley, D., J. Hinderer, G. Casula, O. Francis, H.T.
Hsu, Y. ImAnishi, G. Jentzsch, J. KäÄRiäInEN, J. Merriam, B. Meurers, J. Neumeyer, B. Richter, K. ShibuYa, T. SATo and T. VAN DAM (1999): Network of superconducting gravimeters benefits a number of disciplines, Eos, 80 (11), 121/125-126.

Dehant, V., P. Defraigne and J. Wahr (1999): Tides for a convective Earth, J. Geophys. Res., 104 (B1), 10351058 .

DuCARME, B. (1970): Sensitivity smoothing before the analysis of tidal data, Bull. Inf. Marées Terrestres, 81, 4962-4981.

Ducarme, B. and A. Somerhausen (1997): Tidal gravity recording at Brussels with a SCINTREX CG3-M gravimeter, Bull. Inf. Marées Terrestres, 126, 96119634.

Ducarme, B., A.P. Venedikov, J. Arnoso and R. Vieira (2004): Determination of the long period tidal waves in the GGP superconducting gravity data, J. Geodyn., 38, 307-324.

Ducarme, B., H.P. Sun and J.Q. Xu (2006): Determination of the free core nutation period from tidal gravity observations of the GGP superconducting gravimeter network, J. Geodesy, 81, 179-187, doi: 10.1007/s00190006-0098-9.

EANES, R. and S. BetTAdPuR (1996): The CSR3.0 global ocean tide model: diurnal and demi-diurnal ocean tides from TOPEX/POSEIDON altimetry, CRS-TM-96-05, (University of Texas, Centre for Space Research, Austin, Texas).

El Wahabi, A., B. Ducarme, M. van Ruymbeke, N. D'Oreyè and A. Somerhausen (1997): Continuous gravity observations at Mt. Etna (Sicily) and correlations between temperature and gravimetric records, Cahiers du Centre Européen de Géodynamique et de Séismologie, 14, 105-119.

El Wahabi, A., B. Ducarme and M. van Ruymbeke (2001): Humidity and temperature effects on LaCoste \& Romberg gravimeters, Proceedings of the XIV Int. Symp. On Earth Tides, J. Geodetic Soc. Japan, 47 (1), 10-15.

FARRELL, W.E. (1972): Deformation of the Earth by surface load, Rev. Geophys., 10, 761-779.

KAngieser, E. and W. Torge (1981): Calibration of LaCoste-Romberg gravity meters, Model G and D, Bull. Inf. Bur. Grav. Int., 49, 50-63.

Le Provost, C., M.L. Genco, F. Lyard, P. Vincent and P. CANCEIL (1994): Spectroscopy of the ocean tides from a finite element hydrodynamic model, J. Geophys. Res., 99 (C12), 24777-24797.

Matsumoto, K., M. Ooe, T. Sato et al. (1995): Ocean tides model obtained from TOPEX/POSEIDON altimeter data, J. Geophys. Res., 100, 25319-25330.

Matsumoto, K., T. TAKANEZAWA and M. OoE (2000): Ocean tide models developed by assimilating TOPEX/POSEIDON altimeter data into hydrodynamical model: a global model and a regional model around Japan, $J$. Oceanogr., 56, 567-581

Melchior, P. (1978): The Tides of the Planet Earth, (Pergamon Press), pp. 609.

Melchior, P. (1994): A new data bank for tidal gravity measurements (DB92), Physics Earth Planet. Int., 82, 125-155.

Melchior, P., M. Moens and B. Ducarme (1980): Compu- 
tations of tidal gravity loading and attraction effects, Bull. Inf. Marées Terrestres, 4 (5), 95-133.

Panepinto, S., F. Greco, D. Luzio and B. Ducarme (2006): An overview on wavelet multi-resolution decomposition compared with traditional frequency domain filtering for continuous gravity data denoising, Bull. Inf. Marées Terrestres, 141, 11213-11223.

RAY, R.D. (1999): A global ocean tide model from TOPEX/POSEIDON altimetry: GOT99, NASA Tech. Mem. 209478 (Goddard Space Flight Centre, Greenbelt, MD, U.S.A.).

SCHWIDERSKI, E.W. (1980): Ocean tides I, global ocean tidal equations, Mar. Geodesy, 3, 161-217.

Shum, C.K., P.L. Woodworth, O.B. ANDERSEN, G. Egbert, O. Francis, C. King, S. Klosko, C. Le Provost, X. Li, J.M. Molines, M. Parke, R. Ray, M. Schlax, D. Stammer, C. Temey, P. Vincent and C. Wunsch (1997): Accuracy assessment of recent ocean tide models, J. Geophys. Res., 102 (C11), 25,173-25,194.

Timmen, L. and H.-G. WenZEL (1994): Improved gravimetric Earth tide parameters for station Hannover, Bull. Inf. Marées Terrestres, 119, 8834-8846.

VAN CAMP, M. and P. VAUTERIN (2005): Tsoft: graphical and interactive software for the analysis of time series and Earth tides, Comput. Geosci., 31, 631-640.

vAN RuYMBEKE, M. (1998): Internal precision of the calibration for the LaCoste \& Romberg gravimeters equipped with a feedback system, in Proceedings of the XIII Int. Sympos. Earth Tides, edited by B. DuCARME and P. PAQueT, July 22-25, 1997, Brussels, Belgium, 59-68.

WARburton, R.J. and J.M. GoodKInd (1977): The influence of barometric-pressure variations on gravity, Geophys. J. R. Astron. Soc., 48, 281-292.

WENZEL, H.G. (1994): Earth tide analysis package ETERNA 3.0., Bull. Inf. Marées Terrestres, 118, 8719-8721.

Wenzel, H.G. (1998): Earth tide data processing package ETERNA 3.30: the nanoGal software, in Proceedings of the XIII Int. Sympos. Earth Tides, edited by B. Ducarme and P. PaqueT, July 22-25, 1997, Brussels, Belgium, 487-494.

WENZEL, H.G., W. ZüRn and T.F. BAKER (1991): In situ calibration of LaCoste-Romberg Earth Tide Gravity meter ET19 at BFO Schiltach, Bull. Inf. Marées Terrestres, 109, 7849-7883.

ZaHRAN, K.H. (2000): Accuracy assessment of Ocean Tide loading computations for precise geodetic observations, Ph.D. Thesis (Universität Hannover). 